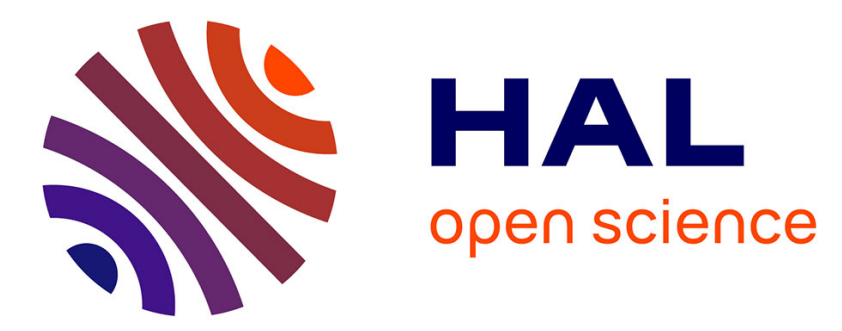

\title{
Unraveling the role of retrieval deficits in autobiographical memory impairment in schizophrenia: A comparison of involuntary and voluntary autobiographical memories
}

Mélissa Allé, Fabrice Berna, Jean-Marie Danion, Dorthe Berntsen

\section{To cite this version:}

Mélissa Allé, Fabrice Berna, Jean-Marie Danion, Dorthe Berntsen. Unraveling the role of retrieval deficits in autobiographical memory impairment in schizophrenia: A comparison of involuntary and voluntary autobiographical memories. Schizophrenia Research, 2021, 228, pp.89-96. 10.1016/j.schres.2020.12.013 . hal-03273694

\section{HAL Id: hal-03273694 \\ https://hal.univ-lille.fr/hal-03273694}

Submitted on 29 Jun 2021

HAL is a multi-disciplinary open access archive for the deposit and dissemination of scientific research documents, whether they are published or not. The documents may come from teaching and research institutions in France or abroad, or from public or private research centers.
L'archive ouverte pluridisciplinaire HAL, est destinée au dépôt et à la diffusion de documents scientifiques de niveau recherche, publiés ou non, émanant des établissements d'enseignement et de recherche français ou étrangers, des laboratoires publics ou privés. 
Unraveling the Role of Retrieval Deficits in Autobiographical Memory Impairment in

Schizophrenia: A Comparison of Involuntary and Voluntary Autobiographical Memories

$$
\text { Mélissa C. Alléa*, Fabrice Berna }{ }^{\mathrm{b}} \text {, Jean-Marie Danion }{ }^{\mathrm{b}} \text {, Dorthe Berntsen }{ }^{\mathrm{a}}
$$

${ }^{a}$ Center on Autobiographical Memory Research, Department of Psychology and Behavioural Sciences, Aarhus University, Denmark, and ${ }^{\mathrm{b}}$ Inserm U1114, Strasbourg University, University Hospital of Strasbourg, France

Text body word count: 4037

Abstract word count: 149

*Corresponding author:

Mélissa C. Allé

Center on Autobiographical Memory Research

Department of Psychology and Behavioural Sciences

Aarhus University

Bartholins Allé 11

8000 Aarhus C

+4587165475

Email: melissa@psy.au.dk 
Running head: Involuntary and Voluntary Recall in Schizophrenia

\begin{abstract}
Patients with schizophrenia show severe autobiographical memory impairment, thought to reflect retrieval deficits caused by executive dysfunction. However, prior research has focused exclusively on strategic (voluntary) retrieval, and ignored involuntary retrieval resulting from automatic and associative processes, involving minimal cognitive control. We report two studies with patients diagnosed with schizophrenia, $(N \mathrm{~s}=40$ and 50 respectively) comparing their impairment in involuntary versus voluntary autobiographical memory. We use two different methodologies, not previously used in schizophrenia research: a naturalistic study involving real-life data and an experimental setup. Both studies consistently showed that involuntary and voluntary autobiographical memories were similarly impaired in schizophrenia. The absence of interaction effects between group and retrieval suggests that schizophrenic patients did not benefit from memory tasks involving little retrieval effort. These findings suggest that autobiographical memory impairment in schizophrenia are not caused by problems with self-initiated voluntary retrieval, but instead likely reflect encoding or binding deficits.
\end{abstract}

Keywords: autobiographical memory, involuntary retrieval, schizophrenia, diary study, executive function 
Running head: Involuntary and Voluntary Recall in Schizophrenia

Unraveling the Role of Retrieval Deficits in Autobiographical Memory Impairment in Schizophrenia: A Comparison of Involuntary and Voluntary Autobiographical Memories

\section{Introduction}

Autobiographical memory is the ability to recollect personal past experiences and contributes to an individual's sense of self(Conway, 2005; Conway and Pleydell-Pearce, 2000). As such, there is a long history of research into autobiographical memory in schizophrenia in order to better understand patients' self-disorders (Allé et al., 2015; Berna et al., 2011, 2016; Raffard et al., 2010b; Ricarte et al., 2017). Overall, previous research has shown that patients with schizophrenia recall fewer autobiographical events than control participants and their memories are less specific, that is, referring to unique events, located in time and place and lasting less than 24 hours (Elvevåg et al., 2003; Riutort et al., 2003). Previous studies have also shown that patients have a reduced capacity for vivid re-experiencing of past events (BennounaGreene et al., 2012; Berna et al., 2016; Cuervo-Lombard et al., 2007; Danion et al., 2005) and that the mental representation of their memories is less detailed than that of control participants (Cuervo-Lombard et al., 2007; Danion et al., 2005). Together these studies point to a severe autobiographical memory impairment in schizophrenia that could account for patients' self disorders (Allé et al., 2015; Berna et al., 2011; Raffard et al., 2010b). One possible explanation of these deficits is that patients have problems with self-initiated, strategic (voluntary) retrieval probably due to executive dysfunction (Danion et al., 2007; Potheegadoo et al., 2014). The executive dysfunction hypothesis is supported by previous studies refuting possible effects of rumination (Ricarte et al., 2014), depression (Berna et al., 2016), and functional avoidance (Harrison \& Fowler, 2004) in retrieval deficits in schizophrenia. The executive dysfunction hypothesis is also at least partly supported by a study showing that patients were able to normalize the richness of details of their memories when a cueing method was used during 
Running head: Involuntary and Voluntary Recall in Schizophrenia

retrieval to help them generating details. However, the same study also showed that some details related to contextual information could not be normalized, suggesting a strong influence of encoding deficits (Potheegadoo et al., 2014).

Importantly, previous studies on autobiographical memory in schizophrenia have focused entirely on voluntarily retrieved memories, although both voluntary and involuntary recalls occur in everyday life, involuntary memories being even more frequent than their voluntary counterpart (Berntsen, 2010). Involuntary autobiographical memories are mental representations of personally experienced past events that come to mind unexpectedly, with no preceding attempt to recall them (Berntsen, 2010, 1998). They result from automatic, associative processes that are outside cognitive control (Hall et al., 2014).

Involuntary and voluntary autobiographical memories appear to originate from the same underlying episodic memory system and share the same basic encoding and maintenance factors (Berntsen, 2010; Hall et al., 2014). However, they differ with respect to how this system is accessed at retrieval (Berntsen et al., 2013; Kompus et al., 2011; Kvavilashvili and Schlagman, 2011), leading to phenomenological differences (Berntsen, 2010). Generally, involuntary autobiographical memories are more specific (Berntsen and Hall, 2004) and have more emotional impact at retrieval (Berntsen and Hall, 2004; Finnbogadóttir and Berntsen, 2011; Rubin et al., 2011, 2008; Watson et al., 2012). Possible explanations for such discrepancies are a different involvement of executive functions at retrieval as well as the influence of cues that facilitate involuntary retrieval. The assumption that involuntary retrieval requires little cognitive effort is supported by brain imaging studies showing that involuntary retrieval takes place with no involvement of prefrontal brain areas generally associated with strategic memory search (Hall et al., 2004), and by experimental studies showing markedly shorter retrieval time for involuntary than voluntary memory (e.g., 
Running head: Involuntary and Voluntary Recall in Schizophrenia

Berntsen, Staugaard \& Sørensen, 2013; Schlagman \& Kvavilashvili, 2008; Staugaard \& Berntsen, 2014).

Because the main difference between involuntary and voluntary autobiographical memories relates to the retrieval phase, comparing the characteristics of involuntarily and voluntarily retrieved memories in patients with schizophrenia will help to clarify the cognitive mechanisms underlying autobiographical memory impairment in this disorder.

Hence, two studies were undertaken with the aim of investigating, for the first time in schizophrenia, the characteristics of involuntary autobiographical memories and comparing them with those of voluntary autobiographical memories. The studies were both designed to provide convergent evidence, using different research methodologies.

Study 1 employed an Ecological Momentary Assessment (EMA; Shiffman et al., 2008) which is a method using repeated collection of real-time data on subjects' behavior and experience in their natural environments (i.e. involuntary autobiographical memories in this context). Using this methodology enabled the collection of critical information about the contexts in which involuntary memories arise in people with schizophrenia in the course of their everyday life, which no other method can supply. It is worth noting, that in schizophrenia, only a very few studies (Berna et al., 2014; Elua et al., 2015; Pernot-Marino et al., 2010; Ricarte et al., 2012) have used this methodology and none in relation to involuntary autobiographical memory.

As a replication and extension, Study 2 was designed to experimentally trigger and assess involuntary and voluntary autobiographical memories in patients with schizophrenia, using a new experimental set up developed especially for this purpose. Study 1 had high ecological validity but was time- and cognitive-demanding for patients. Study 2 was undertaken to address limitations of Study 1 and replicate and extend its findings. First, the experimental design of Study 2 reduced the cognitive effort required to report involuntary 
Running head: Involuntary and Voluntary Recall in Schizophrenia

memories, compared with the diary procedure used in Study 1. The task composing Study 2 was less demanding for participants in terms of motivation and autonomy. Besides, the presence of the experimenter during the task made possible a real-time control of the participants' understanding and completion of the questionnaires. We had two central hypotheses, reflecting different theoretical positions. First, if autobiographical memory impairment in schizophrenia is due to retrieval deficits, patients' memory impairment should be less pronounced or even absent for involuntary autobiographical memories when compared with voluntary autobiographical memories. Second, if patients' autobiographical memory deficit is related to encoding impairment, one could expect similar impairment in patients' involuntary and voluntary memories, as they both rely on the same encoding processes and maintenance factors (Berntsen, 2010).

\section{Study 1}

\section{Methods}

\section{Participants.}

Thirty outpatients with schizophrenia (eight women and 22 men) from the Psychiatry Department of Strasbourg University Hospitals (France) took part in the study. They met the DSM-5(American Psychiatric Association, 2013) criteria for schizophrenia spectrum disorder and, in the preceding three months, they had not experienced any change in their symptoms or medication, or been hospitalized. Symptoms of schizophrenia were assessed by a psychiatrist using the Positive And Negative Syndrome Scale (Kay et al., 1987). All patients were receiving long-term, second-generation antipsychotic treatment. A group of 23 healthy control participants was matched to patients with respect to age, gender and level of schooling (see Table 1, upper part). None of the control participants had a psychiatric illness or was taking 
Running head: Involuntary and Voluntary Recall in Schizophrenia

medication. No participants from either group had a history of neurological disorders or substance abuse. Control participants who scored higher than 8 on the Beck Depression Inventory (Beck et al., 1996) and patients scoring higher than 4 on the Calgary Depression Scale for Schizophrenia (Addington et al., 1993) were excluded from the study. The Sud Méditerranée IV Ethics Committee approved the study (ID-RCB: 2017-A00316-47; ref CPP 1707 03), and all participants gave written, informed consent after the procedures had been fully explained to them. All participants who completed the study were compensated for their participation.

\section{Materials.}

Memory diary. A well-established method of collecting involuntary and voluntary autobiographical memories was employed in the current study (Berntsen and Hall, 2004). This method has been successfully used to discriminate involuntary from voluntary memories in clinical groups, such as in individuals diagnosed with depression (Watson et al., 2012) or PTSD (Rubin et al., 2011). The participants were instructed individually to record 15 involuntary and 15 voluntary memories over the course of several days. Explanations of involuntary and voluntary memories differences, as well as examples, were provided to each participant prior to the onset of the study (see for instance, Berntsen and Hall, 2004).

Involuntary autobiographical memory collection. The first step consisted of recording involuntary memories in a small notebook that was to be carried at all times. Participants were instructed to record information only about the first two spontaneous memories that occurred on a given day. Participants were asked to write down brief details of the memory and to answer questions assessing the characteristics of their autobiographical memory (i.e. memory specificity, vividness, context, and feeling of reliving), using five-point Likert scales

immediately after a memory had occurred (see Table 2). These key properties of autobiographical memory were selected based on the literature as they represent features 
Running head: Involuntary and Voluntary Recall in Schizophrenia

typically associated with the recollective qualities of autobiographical memories (Brewer, 1996; Rubin and Umanath, 2015; Wheeler et al., 1997). There was no requirement to record involuntary memories every day.

The second step was to complete a more detailed questionnaire about their involuntary memories, later in the day on which the memory occurred, supported by the records in the notebook. Participants had to record responses to a series of questions meant to explore characteristics of the memory in relation to hallucination symptoms (Allé et al., 2019, 2018). Of these additional questions, only the spontaneous rehearsal and memory coherence are relevant for the present article (see Table 2). The analysis of other qualitative characteristics is reported elsewhere (Allé et al., 2020).

Voluntary autobiographical memory collection. Immediately after completing the involuntary memory questionnaire, the last step of the procedure consisted of generating a voluntary memory in response to the cue word. The word cues employed were working, snow, sport, school, happy, book, friends, biking, shirt, family, rain, cinema, traveling, phone and car (Berntsen and Hall, 2004). Participants then answered the same series of questions as before, but about the voluntary memory they had just retrieved (see Table 2).

Participants received a reminder phone call about the study every two weeks until they had completed the diary. This was done to motivate them to continue the study and to verify that participants, patients in particular, were doing well and/or to resolve any difficulties they might experience in relation to the study. A debriefing of the study was conducted with each patient after the completion of the protocol. The aim of this debriefing was to collect patients' feedback on the protocol and ensure that none of them experienced side effects as a result of participating in the study.

Coding of memory specificity. Participants assessed the specificity of their own memories. However, this variable was also coded by independent raters as we considered 
Running head: Involuntary and Voluntary Recall in Schizophrenia

specificity an important concept, but difficult for patients to grasp and because the questions were completed at home, with no experimenter present. The experimenter and an independent rater categorized the memories as specific (e.g. a unique event that took place at a particular time in a particular location and lasted less than 24 hours; Williams and Broadbent, 1986; score $=1)$ or non-specific $($ score $=0)$. Inter-rater reliability was good $(\mathrm{K}=0.76)$. Cases with disagreements were resolved by discussion.

\section{Results}

\section{Protocol Completion.}

Only 20 of the 30 patients who began the diary protocol completed it in full. Ten patients gave up the protocol whereas we lost touch with only three control participants. In other words, there were three times as many drop-outs in the patient group as in the control group. The main difficulties and complaints reported by the patients were 1) the lack of motivation and autonomy to complete the diary at home over a period of several weeks, 2) a focus on potentially unpleasant involuntary autobiographical memories and 3) metacognitive difficulties that might have affected diary completion.

\section{Characteristics of Involuntary and Voluntary Memories.}

We compared involuntary and voluntary autobiographical memories in patients with schizophrenia and control participants using a series of two-by-two analyses of variance with group (patients with schizophrenia versus control participants) as a between-subjects variable and type of retrieval (involuntary versus voluntary) as a within-subjects variable (see Table 3, upper part). The dependent variables were mean values, calculated across memories for each participant, or the proportion of memories retrieved per participant. All 32 variables, based on aggregated scores, were normally distributed (Shapiro-Wilk tests >0.96; ps >.16). 
Running head: Involuntary and Voluntary Recall in Schizophrenia

First, there were several main effects of retrieval condition, showing that, compared with voluntary memories, involuntary autobiographical memories were more specific (when externally rated), $F(1,38)=4.39 ; p=.04 ; \eta_{p}^{2}=.05$, less vivid, $F(1,38)=10.63 ; p=.002 ; \eta_{p}^{2}=$ .22 , less contextualized, $F(1,38)=6.72 ; p=.01 ; \eta_{p}{ }^{2}=.15$, and more associated with spontaneous rehearsal, $F(1,38)=13.47 ; p<.001 ; \eta_{p}{ }^{2}=.27$. This shows that involuntary and voluntary memories differed with regard to their qualitative characteristics, and thus, that the present diary study was sufficiently sensitive to capture differences between the two types of retrieval.

Several main effects of group were observed, indicating differences between patients' and controls' autobiographical memories. First, there was group effect for contextual information, $F(1,38)=10.73 ; p=.002 ; \eta_{p}{ }^{2}=.22$, showing that patients retrieved less contextual information than controls in the cases of both involuntary and voluntary retrieval. Second, patients' memories evoked fewer feelings of reliving the experience, $F(1,38)=4.15$; $p=.048 ; y_{p}{ }^{2}=.10$ than those of controls. A trend was also observed for memory specificity, $F(1,48)=3.24 ; p=.06 ; \eta_{p}^{2}=.08$, suggesting that patients' memories tended to be less specific than those of controls. Importantly, there was no significant interaction between group and type of retrieval, for any variable (all $p s>.23 ; \eta_{p}{ }^{2} s<.03$ ).

\section{Discussion}

Study 1 showed that involuntary and voluntary autobiographical memories were similarly impaired in schizophrenia, with respect to both contextual information and the feeling of reliving. The absence of interaction effect between group and type of retrieval suggests that involuntary memories are as impaired as their voluntary counterparts in these patients. These results contradict the hypothesis of a retrieval deficit in schizophrenia being responsible for autobiographical memory impairments in schizophrenia and support an alternative explanation based on encoding or binding deficits. 
Running head: Involuntary and Voluntary Recall in Schizophrenia

The present study replicated some of the effects of involuntary versus voluntary retrieval on memory characteristics (for memory specificity and spontaneous rehearsal) reported in the literature on non-clinical populations (Berntsen, 2009; Berntsen and Jacobsen, 2008; Johannessen and Berntsen, 2010). However, some variables usually differing between involuntary and voluntary autobiographical memory showed unexpected patterns (e.g. memory vividness being higher for voluntary memories compared to involuntary memories). These observations confirm, to some extent, that our diary protocol was an effective method for collecting and differentiating between involuntary and voluntary autobiographical memories, in the current sample of patients with schizophrenia and healthy controls. Important advantages of the diary method include that memories are reported and rated immediately rather than retrospectively. Involuntary memories were collected as they arose during participants' daily life; not taxing participants by requiring them to report numerous memories on the same day and minimizing selection bias by only collecting information about the first two memories of the day (Berntsen, 2009). However, because of its complexity, Study 1 has a limited sample size. Like other studies that have used diaries to collect data on everyday cognition in schizophrenia (Berna et al., 2014; Elua et al., 2015; Pernot-Marino et al., 2010), patients reported difficulties in completing the protocol, and this is reflected in the high proportion of patients who gave up (30\%). Some of their difficulties, mentioned in the results section, might be related to patients' broader metacognitive deficits (Arnon-Ribenfeld et al., 2017). In addition, it is most likely that only patients relatively well functioning, that is non-severe cognitive disorders, were able to complete the study.

Taking into consideration patients' difficulties in completing the diary at home and the unusual pattern of certain memory characteristics, we developed a new experimental setup designed to facilitate patients' participation and to ensure the proper understanding and completion of the questionnaires. 
Running head: Involuntary and Voluntary Recall in Schizophrenia

\section{Study 2}

Study 2 was undertaken to replicate Study 1 while reducing some of its limitations. Hence, we developed a new experimental paradigm designed to elicit involuntary autobiographical memories and discriminate them from voluntary autobiographical memories, in a controlled environment.

\section{Methods}

\section{Participants.}

Twenty-five outpatients with schizophrenia (seven women and 18 men) from the Psychiatry Department of Strasbourg University Hospitals (France) took part in the study, as well as a group of 25 matched control participants (see Table 1, lower part). Patients' and control participants' recruitment was based on the same criteria as in Study 1. Six patients out of the 25 patients also participated in Study 1.

\section{Design.}

The design was a 2 (Group: patients with schizophrenia vs. control participants) $\times 2$ (Retrieval: involuntary vs. voluntary) mixed design with Group as a between groups variable and Retrieval as within-subject variables.

\section{Materials.}

A computerized task was designed in E-Prime 2.0 Professional (Psychology Software Tools), consisting of two distinct sessions: the involuntary autobiographical memory elicitation and the voluntary autobiographical memory collection.

Two series of words or phrases, previously recorded by the experimenter and orally presented via a laptop, were used as cues, either to elicit involuntary autobiographical 
Running head: Involuntary and Voluntary Recall in Schizophrenia

memory in Session 1 or to sample voluntary autobiographical memories in Session 2. The series of words were composed of 80 items each, based on words previously used by Berntsen et al. (1998), and included various semantic categories (activities, objects, sensory experiences, generic - non personal - themes, life themes, places, wordings, feelings). The two series were randomly assigned to the two different sessions.

\section{Procedure.}

Each participant was individually tested at the psychiatric hospital of Strasbourg, France. The two sessions took place at two different days (separated by a maximum of seven days) and were always administered in the same order to avoid the contamination of the involuntary autobiographical memory elicitation with the voluntary autobiographical memory task.

Participants were seated in front of a laptop displaying a black screen. Each series of words or phrases was played aloud.

Involuntary autobiographical memory elicitation. During the first session, participants were presented the series of words, with a new word presented every five seconds, and instructed to do nothing else but listening carefully to those words. If, at some point, they thought about a past situation during the task, they had to press the spacebar to interrupt the task. The button press aimed to record retrieval time for each involuntary memory elicited. Immediately after the task interruption, a written description of the memory was collected. When ten involuntary autobiographical memories had been elicited, participants self-rated the same memory characteristics as in Study 1, using 5-point Likert scales (see Table 2).

Voluntary autobiographical memory collection. The second session consisted in exactly the same setup except that participants were specifically asked to strategically retrieve a memory in relation to each word or phrase orally presented. In this condition, the series of 
Running head: Involuntary and Voluntary Recall in Schizophrenia

words was presented with a 60 -second delay between each word, to give participants time to think about a personal event and find a memory. Similarly to the first session, participants were told to immediately report when they remembered a past event, by pressing the spacebar. Once 10 voluntary memories were collected, participants were asked to answer the same questions as in the involuntary condition to assess the characteristics of their voluntary autobiographical memories (see Table 2).

\section{Results}

We compared the characteristics of involuntary and voluntary autobiographical memories in patients with schizophrenia and control participants using a series of two-by-two analyses of variance with group (patients with schizophrenia versus control participants) as a between-subjects variable and type of retrieval (involuntary versus voluntary) as a withinsubjects variable (see Table 3, lower part). The dependent variables were mean values, calculated across memories for each participant, or the proportion of memories retrieved per participant. All but three (of the 32) variables, based on aggregated scores, were normally distributed (Shapiro-Wilk tests $>0.95 ; p s>.11$ ). In the involuntary condition, mean scores of context, vividness and reliving were not normally distributed (Shapiro-Wilk tests < 0.94; ps $<.02)$.

\section{Experimental validity: effects of voluntary versus involuntary retrieval}

We examined how involuntary retrieval differs from voluntary retrieval, in order to validate the new paradigm we developed.

Characteristics of autobiographical memory retrieval (see Table 4). First, the number of memories elicited was significantly higher in the voluntary condition compared to the involuntary condition, $F(1,48)=29.88 ; p<.001 ; \eta_{p}{ }^{2}=.39$. Second, the number of cues (i.e. words or phrases) necessary to elicit autobiographical memories was significantly higher in 
Running head: Involuntary and Voluntary Recall in Schizophrenia

the involuntary condition than in the voluntary condition, $F(1,48)=383.34 ; p<.001 ; \eta_{p}{ }^{2}=$ .89 , reflecting that involuntary memories required, on average, five times more cues to be elicited than voluntary memories. Third, the retrieval time was significantly higher in the voluntary condition, and participants also assessed their cognitive effort associated with memory retrieval as being higher in the voluntary condition compared to the involuntary condition (Table 4), respectively, $F(1,48)=40.32 ; p<.001 ; \eta p^{2}=.46$, and $F(1,48)=63.17 ; p$ $<.001 ; \eta_{p}{ }^{2}=.58$. Those retrieval differences show that current experimental paradigm reliably distinguished involuntary from voluntary memories.

Self-rated qualitative characteristics of autobiographical memory. Vividness, contextual information and spontaneous rehearsal were higher in involuntary autobiographical memories in comparison to voluntary autobiographical memories, $F s(1,48)>6.34$; ps $<.02$; $\eta_{p}{ }^{2}>.12$ (see Table 3, lower part).

\section{Effects of patient versus control group and interaction effects.}

Characteristics of autobiographical memory retrieval (see Table 3, lower part). Significant group effects were observed for the number of cues and the cognitive effort associated with retrieval, $F s(1,48)>4.72 ; p s<.03 ; \eta_{p}{ }^{2}>.09$. Patients needed fewer cues than control participants to retrieve the same number of memories, but patients self-rated their cognitive effort at retrieval higher in comparison to control participants.

Interaction effects were observed between group and retrieval for the number of memories collected, $F(1,48)=4.18 ; p=.047 ; \eta_{p}^{2}=.08$, and the number of cues used to generate them, $F(1,48)=5.16 ; p=.03 ; y p^{2}=.10$ (Table 4). Post-hoc analyses showed that in the voluntary condition, patients and control participants retrieved the same number of memories $(p=.48)$, using the same number of triggers $(p=.98)$. However, in the involuntary condition, patients tended to report more memories than control participants $(p=.058)$, with fewer triggers $(p=.002)$, see Table 4 for descriptive statistics. 
Running head: Involuntary and Voluntary Recall in Schizophrenia

Self-rated qualitative characteristics of autobiographical memory. Several main effects of group were observed, showing a consistent pattern with patients' memories characteristics being rated lower than those of control participants (see Table 3, lower part). We observed significant group effects for the mean ratings of contextual information, the feeling of reliving, the vividness and the coherence, $F s(1,48)>4.63 ; p s<.04 ; \eta_{p}{ }^{2}>.09$, reflecting that patients' memories were less contextualized and less associated with a feeling of reliving, less vivid and less coherent compared with controls' memories. Trends were also observed for memory specificity and spontaneous rehearsal, $F s(1,48)>3.38$; $p s<.07 ; \eta_{p}{ }^{2}>$ .07 , suggesting that patients' memories tended to be less specific but more often

spontaneously rehearsed. No significant interaction between group and type of retrieval was observed, for any qualitative characteristics (all $p s>.14 ; \eta_{p}{ }^{2}<.04$ ).

\section{Discussion}

Compared with Study 1, the task used Study 2 seemed to be easier to accomplish for patients with schizophrenia, as all of the patients completed the study. Study 2 replicated findings in Study 1 by showing that involuntary and voluntary autobiographical memories were similarly impaired in schizophrenia with respect to both contextual information and the feeling of reliving. In addition, Study 2 extended these results to other phenomenological characteristics of autobiographical memory: vividness and coherence. Similar impairment of both voluntary and involuntary autobiographical memories is a further argument in favor of encoding deficits in schizophrenia.

\section{General Discussion}

We reported two studies that aimed to shed light on the role of retrieval deficits in autobiographical memory impairments in schizophrenia. The diary method, used in Study 1, enabled the collection of a unique set of data on spontaneous autobiographical memories from 
Running head: Involuntary and Voluntary Recall in Schizophrenia

patients with schizophrenia daily life. The experimental paradigm introduced in Study 2 largely replicated Study 1 in a different sample of participants, using another type of methodology. Study 2 reduced some of the limitations in Study 1, and enabled us to better control participants' understanding and completion of the questionnaires. Both studies are novel, and together provide important convergent evidence against retrieval deficits being the primary cause for autobiographical memory impairment in schizophrenia.

Several differences were observed between patients' and controls' autobiographical memories. First, in both studies, the contextual information and the feeling of reliving, were lower in patients' autobiographical memories. Also, both studies showed a trend for specificity to be reduced in the patient group. Second, Study 2 showed several further impairments in patients' memory, affecting memory vividness, coherence, and spontaneous rehearsal. The current results are in full agreement with existing literature on voluntary autobiographical memory in schizophrenia (Berna et al., 2016; Danion et al., 2005; Raffard et al., 2010a), and for the first time, extend these findings to involuntary autobiographical memory.

Importantly, with regard to the qualitative memory characteristics, no interactions between type of retrieval and group were observed. These findings, consistent across both studies and both methodologies, show that involuntary memories are as impaired as their voluntary counterparts in schizophrenia. Considering the fact that involuntary memories occur with no or very little preceding retrieval effort (e.g., Hall et al., 2014; Schlagman \& Kvavilashvili, 2008), our results rule out problems with self-initiated strategic retrieval as a central cause for autobiographical memory deficit in schizophrenia.

An absence of differential group effects of the retrieval manipulation suggests that either encoding deficit is responsible for the memory impairment observed in schizophrenia, or that a general binding impairment affects both memory encoding and retrieval in 
Running head: Involuntary and Voluntary Recall in Schizophrenia

schizophrenia. Supporting this second hypothesis, neuroimaging studies have showed that long-term episodic memory deficits in schizophrenia were associated with abnormal recruitment of the hippocampus (Herbener, 2009; Ongür et al., 2006), which contributes to scene construction through the processing of spatial information and the binding of disparate elements into a coherent scene (Addis and Schacter, 2008; Hassabis et al., 2007). In addition, patients with schizophrenia have been found to have severe difficulty in integrating peripheral features into a coherent representation of a scene in working memory (Bazin et al., 2000; Cohen et al., 1999), and in identifying source and destination memory (Brébion et al., 2002; El Haj et al., 2017). This deficit could hinder the formation of an integrated representations of the current situation or scene (Altamura et al., 2013; Danion et al., 1999), thus preventing patients from experiencing coherent mental time travel later on (Rubin et al., 2019).

Several potential mechanisms can account for a putative encoding deficit in schizophrenia. First, patients with schizophrenia suffer from various well-described perceptual deficits (Kogata and Iidaka, 2018) that could affect the information encoded and account for the subsequent impairment in retrieval that is observed in schizophrenia. In the study by Potheedagoo et al. (2014) the specific cueing procedure was able to "normalize" patients' performance for some categories of details (cognitive and emotional) but did not alter the performance for other categories of details, such as contextual and sensoryperceptual details. This tentatively suggests that the latter details might have been poorly encoded (so that improving retrieval has insufficient effect on performance).

Other health-related factors could also mediate the deficits in autobiographical memory remembering in schizophrenia, such as sleep disorders (Delhikar et al., 2019), fatigue or body mass index (Cheke et al., 2016) but are beyond the scope of the present studies.

In conclusion, the present studies showed that involuntary and voluntary autobiographical memory are similarly impaired in schizophrenia with regard to several 
Running head: Involuntary and Voluntary Recall in Schizophrenia

phenomenological characteristics. These findings are likely to provide new insights into the cognitive mechanisms underlying the autobiographical memory impairment in schizophrenia and tend to support the hypothesis of a general binding impairment or encoding deficit in those patients. Further research should test the assumption of an encoding deficit being responsible for autobiographical memory deficit in schizophrenia, for example by experimentally manipulating scene complexity during encoding.

The present results suggest the relevance of introducing therapies that specifically target encoding difficulties in patients with schizophrenia. For instance, teaching participants encoding strategies enhancing binding has been shown to reduce memory deficit in clinical populations by at encoding (Corrêa et al., 2015). The use of new technologies, such as wearable cameras (Allé et al., 2017; Silva et al., 2013), also have the potential to strengthen re-encoding of autobiographical events via exposure to the photographs taken by the device (Woodberry et al., 2015) and may therefore be especially useful for patients with schizophrenia (Dassing et al., 2020). 
Running head: Involuntary and Voluntary Recall in Schizophrenia

\section{References}

Addington, D., Addington, J., Maticka-Tyndale, E., 1993. Assessing depression in schizophrenia: the Calgary Depression Scale. Br. J. Psychiatry. Suppl. 39-44.

Addis, D.R., Schacter, D.L., 2008. Constructive episodic simulation: temporal distance and detail of past and future events modulate hippocampal engagement. Hippocampus 18, $227-237$.

https://doi.org/10.1002/hipo.20405

Allé, M.C., Berna, F., Berntsen, D., 2019. Individuals with psychotic-like experiences exhibit enhanced involuntary autobiographical memories. Psychiatry Res. 273, 281-287.

https://doi.org/10.1016/j.psychres.2019.01.050

Allé, M.C., Berna, F., Berntsen, D., 2018. Involuntary Autobiographical Memory and Future Thought Predicting Hallucination Proneness. Clin. Psychol. Sci. 2167702618785618.

https://doi.org/10.1177/2167702618785618

Allé, M.C., Berna, F., Danion, J.-M., Berntsen, D., 2020. Involuntary Autobiographical Memories in Schizophrenia: Characteristics and Conditions of Elicitation. Front. Psychiatry 11.

https://doi.org/10.3389/fpsyt.2020.567189

Allé, M.C., Manning, L., Potheegadoo, J., Coutelle, R., Danion, J.-M., Berna, F., 2017. Wearable Cameras Are Useful Tools to Investigate and Remediate Autobiographical Memory Impairment: A Systematic PRISMA Review. Neuropsychol. Rev. 27, 81-99. https://doi.org/10.1007/s11065-0169337-x

Allé, M.C., Potheegadoo, J., Köber, C., Schneider, P., Coutelle, R., Habermas, T., Danion, J.-M., Berna, F., 2015. Impaired coherence of life narratives of patients with schizophrenia. Sci. Rep. 5, 12934. https://doi.org/10.1038/srep12934

Altamura, M., Padalino, F.A., Mammarella, N., Fairfield, B., Balzotti, A., Di Domenico, A., Frisullo, E., Bellomo, A., 2013. Are all forms of feature binding disturbed in schizophrenia? Evidence from a central vs. peripheral distinction in working memory. Psychiatry Res. 209, 9-14.

https://doi.org/10.1016/j.psychres.2012.11.015

American Psychiatric Association, 2013. Diagnostic and Statistical Manual of Mental Disorders, Fifth Edition. ed. American Psychiatric Association. https://doi.org/10.1176/appi.books.9780890425596

Arnon-Ribenfeld, N., Hasson-Ohayon, I., Lavidor, M., Atzil-Slonim, D., Lysaker, P.H., 2017. The association between metacognitive abilities and outcome measures among people with schizophrenia: A meta-analysis. Eur. Psychiatry J. Assoc. Eur. Psychiatr. 46, 33-41.

https://doi.org/10.1016/j.eurpsy.2017.08.002

Bazin, N., Perruchet, P., Hardy-Bayle, M.C., Feline, A., 2000. Context-dependent information processing in patients with schizophrenia. Schizophr. Res. 45, 93-101.

Beck, A.T., Steer, R.A., Ball, R., Ranieri, W., 1996. Comparison of Beck Depression Inventories -IA and -II in psychiatric outpatients. J. Pers. Assess. 67, 588-597.

https://doi.org/10.1207/s15327752jpa6703_13

Bennouna-Greene, M., Berna, F., Conway, M.A., Rathbone, C.J., Vidailhet, P., Danion, J.-M., 2012. 
Running head: Involuntary and Voluntary Recall in Schizophrenia

Self-images and related autobiographical memories in schizophrenia. Conscious. Cogn. 21, 247-257. https://doi.org/10.1016/j.concog.2011.10.006

Berna, F., Bennouna-Greene, M., Potheegadoo, J., Verry, P., Conway, M.A., Danion, J.-M., 2011. Impaired ability to give a meaning to personally significant events in patients with schizophrenia. Conscious. Cogn. 20, 703-711. https://doi.org/10.1016/j.concog.2010.12.004

Berna, F., Huron, C., Kazès, M., Offerlin-Meyer, I., Willard, D., Verry, P., Hedélin, G., Krebs, M.-O., Danion, J.-M., 2014. Chronic persecutory delusion and autobiographical memories in patients with schizophrenia: a diary study. Isr. J. Psychiatry Relat. Sci. 51, 25-33.

Berna, F., Potheegadoo, J., Aouadi, I., Ricarte, J.J., Allé, M.C., Coutelle, R., Boyer, L., CuervoLombard, C.V., Danion, J.-M., 2016. A Meta-Analysis of Autobiographical Memory Studies in Schizophrenia Spectrum Disorder. Schizophr. Bull. 42, 56-66. https://doi.org/10.1093/schbul/sbv099

Berntsen, D., 2010. The Unbidden Past: Involuntary Autobiographical Memories as a Basic Mode of Remembering. Curr. Dir. Psychol. Sci. 19, 138-142. https://doi.org/10.1177/0963721410370301

Berntsen, D., 2009. Involuntary Autobiographical Memories: An Introduction to the Unbidden Past. Cambridge University Press.

Berntsen, D., 1998. Voluntary and involuntary access to autobiographical memory. Mem. Hove Engl. 6, 113-141. https://doi.org/10.1080/741942071

Berntsen, D., Hall, N.M., 2004. The episodic nature of involuntary autobiographical memories. Mem. Cognit. 32, 789-803.

Berntsen, D., Jacobsen, A.S., 2008. Involuntary (spontaneous) mental time travel into the past and future. Conscious. Cogn. 17, 1093-1104. https://doi.org/10.1016/j.concog.2008.03.001

Berntsen, D., Staugaard, S.R., Sørensen, L.M.T., 2013. Why am I remembering this now? Predicting the occurrence of involuntary (spontaneous) episodic memories. J. Exp. Psychol. Gen. 142, 426-444. https://doi.org/10.1037/a0029128

Brébion, G., Gorman, J.M., Amador, X., Malaspina, D., Sharif, Z., 2002. Source monitoring impairments in schizophrenia: characterisation and associations with positive and negative symptomatology. Psychiatry Res. 112, 27-39.

Brewer, W.F., 1996. What is recollective memory?, in: Remembering Our Past: Studies in Autobiographical Memory. Cambridge University Press, New York, NY, US, pp. 19-66. https://doi.org/10.1017/CBO9780511527913.002

Cheke, L.G., Simons, J.S., Clayton, N.S., 2016. Higher body mass index is associated with episodic memory deficits in young adults. Q. J. Exp. Psychol. 2006 69, 2305-2316.

https://doi.org/10.1080/17470218.2015.1099163

Cohen, J.D., Barch, D.M., Carter, C., Servan-Schreiber, D., 1999. Context-processing deficits in schizophrenia: converging evidence from three theoretically motivated cognitive tasks. J. Abnorm. Psychol. 108, 120-133.

Conway, M.A., 2005. Memory and the Self. J. Mem. Lang. 53, 594-628. 
Running head: Involuntary and Voluntary Recall in Schizophrenia

Conway, M.A., Pleydell-Pearce, C.W., 2000. The construction of autobiographical memories in the self-memory system. Psychol. Rev. 107, 261-288.

Corrêa, M.S., da Silveira, E.M.S., de Lima, D.B., Balardin, J.B., Walz, J.C., Kapczinski, F., Bromberg, E., 2015. The role of encoding strategies in contextual memory deficits in patients with bipolar disorder. Neuropsychol. Rehabil. 25, 122-136. https://doi.org/10.1080/09602011.2014.969281

Cuervo-Lombard, C., Jovenin, N., Hedelin, G., Rizzo-Peter, L., Conway, M.A., Danion, J.-M., 2007. Autobiographical memory of adolescence and early adulthood events: an investigation in schizophrenia. J. Int. Neuropsychol. Soc. JINS 13, 335-343.

https://doi.org/10.1017/S135561770707035X

Danion J, Rizzo L, Bruant A, 1999. FUnctional mechanisms underlying impaired recognition memory and conscious awareness in patients with schizophrenia. Arch. Gen. Psychiatry 56, 639-644. https://doi.org/10.1001/archpsyc.56.7.639

Danion, J.-M., Cuervo, C., Piolino, P., Huron, C., Riutort, M., Peretti, C.S., Eustache, F., 2005. Conscious recollection in autobiographical memory: an investigation in schizophrenia. Conscious. Cogn. 14, 535-547. https://doi.org/10.1016/j.concog.2005.01.005

Danion, J.-M., Huron, C., Vidailhet, P., Berna, F., 2007. Functional mechanisms of episodic memory impairment in schizophrenia. Can. J. Psychiatry Rev. Can. Psychiatr. 52, 693-701.

Dassing, R., Allé, M.C., Cerbai, M., Obrecht, A., Meyer, N., Vidailhet, P., Danion, J.-M., Mengin, A.C., Berna, F., 2020. Cognitive Intervention Targeting Autobiographical Memory Impairment in Patients With Schizophrenia Using a Wearable Camera: A Proof-of-Concept Study. Front. Psychiatry 11. https://doi.org/10.3389/fpsyt.2020.00397

Delhikar, N., Sommers, L., Rayner, G., Schembri, R., Robinson, S.R., Wilson, S., Jackson, M.L., 2019. Autobiographical Memory From Different Life Stages in Individuals With Obstructive Sleep Apnea. J. Int. Neuropsychol. Soc. JINS 25, 266-274. https://doi.org/10.1017/S1355617718001091

El Haj, M., Altman, R., Bortolon, C., Capdevielle, D., Raffard, S., 2017. Destination memory in schizophrenia: “Did I told Elvis Presley about the thief?” Psychiatry Res. 248, 71-76. https://doi.org/10.1016/j.psychres.2016.12.023

Elua, I., Laws, K.R., Kvavilashvili, L., 2015. Increased frequency of involuntary semantic memories or mind-pops in schizophrenia: a diary study. Cognit. Neuropsychiatry 20, 502-511. https://doi.org/10.1080/13546805.2015.1092431

Elvevåg, B., Kerbs, K.M., Malley, J.D., Seeley, E., Goldberg, T.E., 2003. Autobiographical memory in schizophrenia: an examination of the distribution of memories. Neuropsychology 17, 402-409.

Finnbogadóttir, H., Berntsen, D., 2011. Involuntary and voluntary mental time travel in high and low worriers. Mem. Hove Engl. 19, 625-640. https://doi.org/10.1080/09658211.2011.595722

Hall, S.A., Rubin, D.C., Miles, A., Davis, S.W., Wing, E.A., Cabeza, R., Berntsen, D., 2014. The Neural Basis of Involuntary Episodic Memories. J. Cogn. Neurosci. 26, 2385-2399. https://doi.org/10.1162/jocn_a_00633

Hassabis, D., Kumaran, D., Vann, S.D., Maguire, E.A., 2007. Patients with hippocampal amnesia 
Running head: Involuntary and Voluntary Recall in Schizophrenia

cannot imagine new experiences. Proc. Natl. Acad. Sci. U. S. A. 104, 1726-1731.

https://doi.org/10.1073/pnas.0610561104

Herbener, E.S., 2009. Impairment in long-term retention of preference conditioning in schizophrenia. Biol. Psychiatry 65, 1086-1090. https://doi.org/10.1016/j.biopsych.2009.01.020

Johannessen, K.B., Berntsen, D., 2010. Current concerns in involuntary and voluntary autobiographical memories. Conscious. Cogn. 19, 847-860.

https://doi.org/10.1016/j.concog.2010.01.009

Kay, S.R., Fiszbein, A., Opler, L.A., 1987. The positive and negative syndrome scale (PANSS) for schizophrenia. Schizophr. Bull. 13, 261-276.

Kogata, T., Iidaka, T., 2018. A review of impaired visual processing and the daily visual world in patients with schizophrenia. Nagoya J. Med. Sci. 80, 317-328.

https://doi.org/10.18999/nagjms.80.3.317

Kompus, K., Eichele, T., Hugdahl, K., Nyberg, L., 2011. Multimodal imaging of incidental retrieval: the low route to memory. J. Cogn. Neurosci. 23, 947-960. https://doi.org/10.1162/jocn.2010.21494

Kvavilashvili, L., Schlagman, S., 2011. Involuntary autobiographical memories in dysphoric mood: a laboratory study. Mem. Hove Engl. 19, 331-345. https://doi.org/10.1080/09658211.2011.568495

Ongür, D., Cullen, T.J., Wolf, D.H., Rohan, M., Barreira, P., Zalesak, M., Heckers, S., 2006. The neural basis of relational memory deficits in schizophrenia. Arch. Gen. Psychiatry 63, 356-365. https://doi.org/10.1001/archpsyc.63.4.356

Pernot-Marino, E., Schuster, C., Hedelin, G., Berna, F., Zimmermann, M.-A., Danion, J.-M., 2010. True and false autobiographical memories in schizophrenia: preliminary results of a diary study. Psychiatry Res. 179, 1-5. https://doi.org/10.1016/j.psychres.2009.11.014

Potheegadoo, J., Cordier, A., Berna, F., Danion, J.-M., 2014. Effectiveness of a specific cueing method for improving autobiographical memory recall in patients with schizophrenia. Schizophr. Res. 152, 229-234. https://doi.org/10.1016/j.schres.2013.10.046

Raffard, S., D’Argembeau, A., Bayard, S., Boulenger, J.-P., Van der Linden, M., 2010a. Scene construction in schizophrenia. Neuropsychology 24, 608-615. https://doi.org/10.1037/a0019113

Raffard, S., D’Argembeau, A., Lardi, C., Bayard, S., Boulenger, J.-P., Van der Linden, M., 2010b. Narrative identity in schizophrenia. Conscious. Cogn. 19, 328-340.

https://doi.org/10.1016/j.concog.2009.10.005

Ricarte, J.J., Hernández-Viadel, J.V., Latorre, J.M., Ros, L., 2012. Effects of event-specific memory training on autobiographical memory retrieval and depressive symptoms in schizophrenic patients. J. Behav. Ther. Exp. Psychiatry 43 Suppl 1, S12-20. https://doi.org/10.1016/j.jbtep.2011.06.001

Ricarte, J.J., Ros, L., Latorre, J.M., Watkins, E., 2017. Mapping autobiographical memory in schizophrenia: Clinical implications. Clin. Psychol. Rev. 51, 96-108.

https://doi.org/10.1016/j.cpr.2016.11.004

Riutort, M., Cuervo, C., Danion, J.-M., Peretti, C.S., Salamé, P., 2003. Reduced levels of specific 
Running head: Involuntary and Voluntary Recall in Schizophrenia

autobiographical memories in schizophrenia. Psychiatry Res. 117, 35-45.

Rubin, D.C., Boals, A., Berntsen, D., 2008. Memory in posttraumatic stress disorder: properties of voluntary and involuntary, traumatic and nontraumatic autobiographical memories in people with and without posttraumatic stress disorder symptoms. J. Exp. Psychol. Gen. 137, 591-614. https://doi.org/10.1037/a0013165

Rubin, D.C., Deffler, S.A., Umanath, S., 2019. Scenes enable a sense of reliving: Implications for autobiographical memory. Cognition 183, 44-56. https://doi.org/10.1016/j.cognition.2018.10.024

Rubin, D.C., Dennis, M.F., Beckham, J.C., 2011. Autobiographical memory for stressful events: the role of autobiographical memory in posttraumatic stress disorder. Conscious. Cogn. 20, 840-856. https://doi.org/10.1016/j.concog.2011.03.015

Rubin, D.C., Umanath, S., 2015. Event memory: A theory of memory for laboratory, autobiographical, and fictional events. Psychol. Rev. 122, 1-23. https://doi.org/10.1037/a0037907

Shiffman, S., Stone, A.A., Hufford, M.R., 2008. Ecological momentary assessment. Annu. Rev. Clin. Psychol. 4, 1-32.

Silva, A.R., Pinho, S., Macedo, L.M., Moulin, C.J., 2013. Benefits of SenseCam review on neuropsychological test performance. Am. J. Prev. Med. 44, 302-307.

https://doi.org/10.1016/j.amepre.2012.11.005

Watson, L.A., Berntsen, D., Kuyken, W., Watkins, E.R., 2012. The characteristics of involuntary and voluntary autobiographical memories in depressed and never depressed individuals. Conscious. Cogn. 21, 1382-1392. https://doi.org/10.1016/j.concog.2012.06.016

Wheeler, M.A., Stuss, D.T., Tulving, E., 1997. Toward a theory of episodic memory: the frontal lobes and autonoetic consciousness. Psychol. Bull. 121, 331-354.

Williams, J.M., Broadbent, K., 1986. Autobiographical memory in suicide attempters. J. Abnorm. Psychol. 95, 144-149. https://doi.org/10.1037/0021-843X.95.2.144

Woodberry, E., Browne, G., Hodges, S., Watson, P., Kapur, N., Woodberry, K., 2015. The use of a wearable camera improves autobiographical memory in patients with Alzheimer's disease. Mem. Hove Engl. 23, 340-349. https://doi.org/10.1080/09658211.2014.886703 
Running head: Involuntary and Voluntary Recall in Schizophrenia

Table 1

Patients with Schizophrenia and Control Participants Demographic Information and Clinical Measures for Study 1 and Study 2

\begin{tabular}{|c|c|c|c|c|c|c|c|}
\hline & \multicolumn{2}{|c|}{$\begin{array}{l}\text { Patients with } \\
\text { Schizophrenia }\end{array}$} & \multicolumn{2}{|c|}{$\begin{array}{c}\text { Control } \\
\text { Participants }\end{array}$} & \multicolumn{3}{|c|}{ Statistics } \\
\hline & $M$ & $S D$ & $M$ & $S D$ & $t(38)$ & $d$ & $95 \%$ IC \\
\hline \multicolumn{8}{|l|}{ Study 1} \\
\hline Age (years) & 38.15 & 9.96 & 37.10 & 10.06 & 0.33 & 0.10 & {$[-5.35,7.46]$} \\
\hline Level of education & 12.40 & 1.79 & 12.80 & 1.88 & -0.69 & -0.22 & {$[-0.40,-1.57]$} \\
\hline CDSS & 1.42 & 1.24 & - & - & - & - & - \\
\hline BDI & - & - & 1.35 & 0.91 & - & - & - \\
\hline PANSS - Positive symptoms & 13.41 & 3.43 & - & - & - & - & - \\
\hline PANSS - Negative & 15.59 & 6.15 & - & - & - & - & - \\
\hline PANSS - General & 23.76 & 6.71 & - & - & - & - & - \\
\hline PANSS - Total & 52.82 & 13.43 & - & - & - & - & - \\
\hline Length of illness & 12.40 & 7.73 & - & - & - & - & - \\
\hline \multicolumn{8}{|l|}{ Study 2} \\
\hline Age (years) & 38.20 & 9.67 & 38.68 & 10.39 & -0.17 & -0.05 & {$[-6.19,5.23]$} \\
\hline Level of schooling & 11.64 & 2.21 & 12.08 & 1.87 & -0.76 & -0.22 & {$[-1.60,0.72]$} \\
\hline CDSS & 1.67 & 1.33 & - & - & - & - & - \\
\hline BDI & - & - & 1.39 & 1.02 & - & - & - \\
\hline PANSS - Positive symptoms & 14.31 & 7.54 & - & - & - & - & - \\
\hline PANSS - Negative symptoms & 18.52 & 6.89 & - & - & - & - & - \\
\hline PANSS - General & 22.84 & 7.66 & - & - & - & - & - \\
\hline PANSS - Total & 55.67 & 25.09 & - & - & - & - & - \\
\hline Length of illness & 14.58 & 7.15 & - & - & - & - & - \\
\hline
\end{tabular}




\section{Table 2}

Questions Included in the Memory Questionnaires for both Study 1 and Study 2

1. [specificity self-rated] Is the memory about a specific situation that happened on a specific day in your past? Yes/No

2. [vividness] How vivid is the memory? From $1=$ Vague to $7=$ Clear and vivid as if $I t$ was experienced again

3. [reliving] While remembering the event, I feel as though I am reliving it. From $1=$ Not at all to $7=$ as if it were happening now

4. [context] Can you remember the setting of the event? From $1=$ Not at all to $7=$ Perfectly

5. [coherence] How is your mental representation of this event? From $1=$ My memory comes in pieces with missing bits to $7=$ My memories comes to me as a coherent story

6. [spontaneous rehearsal] This memory has previously come to me "out of the blue", without me trying to think about it. From $1=$ Not at all to $7=$ Very Often

\section{Additional question - Study 2}

[cognitive effort] To which extent have you actively tried to bring the memory to mind? From $1=$ I wasn't trying at all to $7=$ I tried very hard 
Table 3

Measures of Involuntary and Voluntary Autobiographical Memory Characteristics in Patients with Schizophrenia and Control Participants

\begin{tabular}{|c|c|c|c|c|c|c|c|c|c|c|c|c|c|c|}
\hline & \multicolumn{4}{|c|}{ Patients with schizophrenia } & \multicolumn{4}{|c|}{ Control participants } & \multicolumn{6}{|c|}{ Statistics $F(1,48)$} \\
\hline & \multicolumn{2}{|c|}{ Involuntary } & \multicolumn{2}{|c|}{ Voluntary } & \multicolumn{2}{|c|}{ Involuntary } & \multicolumn{2}{|c|}{ Voluntary } & \multicolumn{2}{|c|}{ Group } & \multicolumn{2}{|c|}{ Retrieval } & \multicolumn{2}{|c|}{ Interaction } \\
\hline & $M$ & $S D$ & $M$ & $S D$ & $M$ & $S D$ & $M$ & $S D$ & $F$ & $\mathrm{y}^{2} \mathrm{p}$ & $F$ & $\mathrm{y}_{\mathrm{p}}^{2}$ & $F$ & $\mathrm{y}^{2} \mathrm{p}$ \\
\hline \multicolumn{15}{|l|}{ Study 1 - Diary method } \\
\hline Specificity $^{\text {a }(\text { self-rated) }}$ & 0.65 & 0.22 & 0.66 & 0.20 & 0.78 & 0.60 & 0.72 & 0.18 & $3.24^{\alpha}$ & .08 & 1.05 & .03 & 0.33 & .01 \\
\hline Specificity (externally rated) & 0.69 & 0.19 & 0.56 & 0.23 & 0.70 & 0.17 & 0.66 & 0.17 & 1.68 & .02 & $4.39 *$ & .05 & 1.47 & .03 \\
\hline Vividness & 3.44 & 0.57 & 3.65 & 0.69 & 3.74 & 0.68 & 3.91 & 0.71 & 2.28 & .05 & $10.63^{* *}$ & .22 & 0.12 & .00 \\
\hline Reliving & 3.39 & 0.64 & 3.39 & 0.67 & 3.75 & 0.66 & 3.86 & 0.69 & $4.15^{*}$ & .10 & 0.78 & .02 & 0.68 & .02 \\
\hline Context & 3.53 & 0.67 & 3.84 & 0.61 & 4.17 & 0.53 & 4.29 & 0.54 & $10.73^{* *}$ & .22 & $6.72 *$ & .15 & 1.23 & .03 \\
\hline Coherence & 3.16 & 0.83 & 3.35 & 0.95 & 3.42 & 0.88 & 3.48 & 0.98 & 0.89 & .01 & 2.21 & .07 & 1.13 & .03 \\
\hline Spontaneous Rehearsal & 2.81 & 0.88 & 2.51 & 0.85 & 2.90 & 0.77 & 2.34 & 0.61 & 0.13 & .00 & $13.47 * * *$ & .27 & 0.45 & .01 \\
\hline \multicolumn{15}{|l|}{ Study 2 - Experimental setup } \\
\hline Specificity $^{\mathrm{a}}$ (self-rated) & 0.70 & 0.25 & 0.75 & 0.24 & 0.86 & 0.17 & 0.79 & 0.21 & $3.72^{\alpha}$ & .07 & 0.06 & .00 & 2.23 & .04 \\
\hline Specificity ${ }^{\mathrm{a}}$ (externally rated) & 0.64 & 0.26 & 0.61 & 0.21 & 0.68 & 0.21 & 0.67 & 0.17 & 1.22 & .02 & 0.26 & .01 & 0.12 & .00 \\
\hline Vividness & 5.17 & 1.06 & 4.93 & 1.06 & 5.98 & 0.68 & 5.42 & 1.01 & $8.28 * *$ & .15 & $10.74 * *$ & .19 & 1.19 & .03 \\
\hline Reliving & 5.06 & 1.15 & 5.11 & 0.87 & 5.73 & 1.02 & 5.50 & 0.97 & $4.63 *$ & .09 & 0.41 & .01 & 0.95 & .02 \\
\hline Context & 5.62 & 1.03 & 5.31 & 0.92 & 6.29 & 0.72 & 5.73 & 1.00 & $6.95 *$ & .13 & $11.17 * *$ & .19 & 1.07 & .02 \\
\hline Coherence & 4.31 & 1.35 & 4.00 & 1.26 & 4.90 & 1.17 & 4.90 & 0.97 & $7.80 * *$ & .14 & 1.08 & .02 & 1.16 & .02 \\
\hline Spontaneous Rehearsal & 3.16 & 1.36 & 2.81 & 1.07 & 3.68 & 1.31 & 3.25 & 1.12 & $3.38^{a}$ & .07 & $6.34 *$ & .12 & 0.42 & .01 \\
\hline
\end{tabular}

${ }^{*} p<.10 ; * p<.05 ; * * p<.01$ 
Table 4

Retrieval characteristics of Involuntary and Voluntary Autobiographical Memories in Patients with Schizophrenia and Control Participants

\begin{tabular}{|c|c|c|c|c|c|c|c|c|c|c|c|c|c|c|}
\hline & \multicolumn{4}{|c|}{ Patients with } & \multicolumn{4}{|c|}{ Control participants } & \multicolumn{6}{|c|}{ Statistics $F(1,48)$} \\
\hline & \multicolumn{2}{|c|}{ Involuntar } & \multicolumn{2}{|c|}{ Voluntary } & \multicolumn{2}{|c|}{ Involunta } & \multicolumn{2}{|c|}{ Voluntar } & \multicolumn{2}{|c|}{ Group } & \multicolumn{2}{|c|}{ Retrieval } & \multicolumn{2}{|c|}{ Interacti } \\
\hline & $M$ & $S D$ & $M$ & $S D$ & $M$ & $S D$ & $M$ & $S D$ & $F$ & $\mathrm{y}$ & $F$ & y & $F$ & y \\
\hline Numb & 8.37 & 2.34 & 9.5 & 1.18 & 7.3 & 2.6 & 9.9 & 0.4 & 0.62 & .0 & $29.88 * *$ & .3 & 4.18 & .0 \\
\hline Retrie & 3.96 & 0.83 & $1 \hat{1}$ & 7.11 & 3.7 & 0.6 & 9.7 & 4.3 & 1.41 & .0 & $63.17 * *$ & .5 & 0.99 & .0 \\
\hline Numb & 60.2 & 21.9 & 12. & 4.82 & 72 . & 15. & $\hat{12 .}$ & 4.1 & $4.72 *$ & .0 & $383.34 *$ & .8 & 5.16 & .1 \\
\hline Cognit & 2.58 & $1 . \hat{6} 1$ & $\begin{array}{c}{ }^{20} \\
3.7\end{array}$ & 1.10 & $\hat{1.7}$ & 0.6 & $\begin{array}{c}\varepsilon_{3} \\
3.0\end{array}$ & 1.2 & $7.22 *$ & .1 & $40.32 * *$ & .4 & 0.29 & .0 \\
\hline
\end{tabular}

